\title{
A Novel Hybrid Coupler Based on Antiresonant Reflecting Optical Waveguides
}

\author{
Jau-Jan Deng, Student Member, IEEE, Member, OSA, and Yang-Tung Huang, Member, IEEE, Member, OSA
}

\begin{abstract}
A novel hybrid coupler based on the antiresonant reflecting optical waveguides (ARROW's) is presented. This device consists of two parallel antiresonant reflecting optical waveguides with a tapered outermost cladding layer. Such a device can provide advantageous features of low radiation loss, low crosstalk at the output end, and compatible core dimensions and indexes with single-mode optical fibers. The staircase approximation and the eigenmode expansion analysis are used to analyze and optimize this device. In our design on a $\mathrm{Si}$-substrate, radiation losses below $0.38 \mathrm{~dB}$ and extinction ratios below $-20 \mathrm{~dB}$ for two waveguide channels are achieved. The beam propagation method (BPM) is also used to verify our design and analysis results.
\end{abstract}

Index Terms-Optical beam splitting, optical coupling, optical power dividers/combiners, optical resonance, optical waveguides.

\section{INTRODUCTION}

B EAM splitters and beam combiners are basic elements to construct various optical circuits. Optical hybrid couplers, which can provided both of these functions in integrated optics, have been proposed and demonstrated [1]-[6]. Such versatile devices are attractive for applications in optical communications, optical signal processing, optical networks, and optical microsensing systems. A conventional waveguide hybrid coupler consists of a symmetric and an asymmetric Yjunctions connected directly. In each Y-junction, there exist two normal modes, even and odd modes, traveling along the propagation direction. For the input symmetric Y-junction, the amplitudes of the fields for both modes in two individual waveguides are the same; on the other hand, in the output asymmetric Y-junction, the field of the even mode is mainly confined in the wide-arm and the odd mode mainly in the narrow-arm, respectively. The branching angles of two $\mathrm{Y}$ junctions are designed to be small enough so that the device is adiabatic. The input conditions of the optical fields will determine the outputs of the wide- and narrow-arms in the asymmetric Y-junction. However, for a conventional hybrid coupler, there exist inherent disadvantages of difficult alignment and low coupling efficiency when connected to optical fibers due to their incompatible core dimensions or indexes. Moreover, the separations between two arms at the input and output ends must be wide enough to reduce the crosstalk

Manuscript received May 12, 1997; revised October 15, 1997. This work was supported by the National Science Council of the Republic of China under Contract NSC86-2221-E-009-040.

The authors are with the Department of Electronics Engineering and Institute of Electronics, National Chiao Tung University, Hsinchu, Taiwan, R.O.C.

Publisher Item Identifier S 0733-8724(98)02585-7. between two waveguide channels, and thus the device length is difficult to reduce.

The antiresonant reflecting optical waveguides (ARROW's) are promising to replace conventional hybrid couplers because of their flexible design rules of core and cladding dimensions [7]. In comparison with conventional waveguides, ARROW waveguides utilize antiresonant reflection as the guiding mechanism instead of total internal reflection. With this structure, ARROW devices can guide waves in low-index cores with large core sizes such that their core indexes and sizes can be compatible with single-mode fiber indexes and diameters, which provide efficient connections with fibers. In addition, the coupling length of a dual ARROW waveguide is not increasing but varies as a periodic function with the increasing waveguide separation such that a remote coupler can be realized [8], [9]. Based on our previous investigation, it is found that the maximum coupling efficiency of a dual parallel ARROW waveguide can be controlled from 100 to $0 \%$ by simply varying the outermost cladding thickness to change their symmetry [10]. In this paper, we present a novel hybrid coupler based on ARROW waveguides to provide the same functions as a conventional hybrid coupler [11]. The outermost cladding layer is tapered from the exact symmetric structure to the extremely asymmetric one, so that the fields of the symmetric and extremely asymmetric sides are similar to those in the input and output $\mathrm{Y}$-junctions of a conventional hybrid coupler. Compatible core dimensions and indexes with single-mode fibers and a large separation thickness can be achieved by utilizing the periodic property of antiresonant conditions. The input and output coupling efficiency are thus significantly improved. In addition, by an optimum design of the outermost cladding thickness at the input and output ends, equal excitations of two normal modes at the input end and a low crosstalk at the output end can be achieved. Moreover, the surface scattering is relieved because only the thickness of the outermost cladding layer is changed along the device and the fields are mainly confined within the cores.

In Section II, the basic structure of an ARROW hybrid coupler and its operation principle are discussed. In Section III, the continuous tapered structure is modeled by dividing them into various segments with small step discontinuities [12]. The characteristic matrix method is applied to obtain the effective indexes, propagation losses, and field distributions of the propagation modes for each segment [9], [10]. The adiabatic invariance condition is verified by analyzing the mode transition of propagation modes using the abovementioned staircase approximation and eigenmode expansion analysis 
[13]. In Section IV, a design example and the simulation results using the eigenmode expansion analysis are given and the beam propagation method (BPM) is used to verify the theory and design [14]-[16]. Finally, a brief conclusion is given in Section V.

\section{BASIC STRUCTURE AND OPERATION PRINCIPLE OF AN ARROW HYBRID COUPLER}

The configuration of an ARROW hybrid coupler $\left(n_{a} / n_{l_{1}}\right.$ $\left./ n_{h_{1}} / n_{g_{1}} / n_{h_{1}} / n_{\text {sep }} / n_{h_{2}} / n_{g_{2}} / n_{h_{2}} / n_{l_{2}} / n_{s}\right)$ is depicted in Fig. 1, which consists of two parallel ARROW waveguides, a gradually tapered upper cladding layer, and a separation layer. The core layers of two ARROW waveguides are with low refractive indexes of $n_{g_{1}}$ and $n_{g_{2}}$, and thickness of $d_{g_{1}}$ and $d_{g_{2}}$, respectively. The upper waveguide is sandwiched between two high-index $\left(n_{h_{1}}\right)$ cladding layers of thickness $d_{h_{1}}$, and atop this upper sandwiched waveguide is a tapered low-index $\left(n_{l_{1}}\right)$ cladding layer of thickness $d_{l_{1}}$. The lower waveguide is sandwiched between two high-index $\left(n_{h_{2}}\right)$ cladding layers of thickness $d_{h_{2}}$, and under this lower sandwiched waveguide is another low-index $\left(n_{l_{2}}\right)$ cladding layer of thickness $d_{l_{2}}$. Between two ARROW waveguides there is a separation cladding layer with an index of $n_{\text {sep }}$ and a thickness of $d_{\text {sep }}$. The upper core index $\left(n_{g_{1}}\right)$ and dimension $\left(d_{g_{1}}\right)$ are flexibly chosen to be compatible with connected single-mode fibers, the core layer of the lower waveguide must satisfy the transverse resonance condition, and its thickness $d_{g_{2}}$ is given as [7]

$$
d_{g_{2}}=\frac{\lambda}{2 n_{g_{2}}}\left[1-\left(\frac{n_{g_{1}}}{n_{g_{2}}}\right)^{2}+\left(\frac{\lambda}{2 n_{g_{2}} d_{g_{1}}}\right)^{2}\right]^{-(1 / 2)} .
$$

Except the upper cladding layer of $d_{l_{1}}$, all cladding layers satisfy the transverse antiresonance condition as

$$
\begin{aligned}
d_{j}= & \frac{\lambda}{4 n_{j}}\left[1-\left(\frac{n_{g_{1}}}{n_{j}}\right)^{2}+\left(\frac{\lambda}{2 n_{j} d_{g_{1}}}\right)^{2}\right]^{-(1 / 2)} \\
& \cdot\left(2 P_{j}+1\right), \quad P_{j}=0,1,2,3, \cdots
\end{aligned}
$$

where $j=h_{1}$ (and $h_{2}$ ), sep, and $l_{2}$, representing corresponding high-index, separation and low-index cladding layers, respectively. $P_{j}$ is the order of antiresonant condition, which can be arbitrarily chosen to adopt to butt-connection with singlemode fibers easily. Since the coupling strength between dual ARROW waveguides strongly depends on the degree of their symmetry, the thickness of the upper cladding layer can be adjusted to change the degree of their symmetry and thus the coupling efficiency between two guides can be varied. In our ARROW hybrid coupler device, the dual waveguides are exactly symmetric at the input end and extremely asymmetric at the output end. The thickness of the upper cladding layer $\left(d_{l_{1}}\right)$ is thus gradually tapered from the input end to the output end as shown in Fig. 1.

Throughout the whole device there are two normal modes (one even and one odd modes) traveling along the propagation direction. The typical field distributions of two normal modes at several corresponding positions along the propagation direction are shown in Fig. 2. The peak amplitudes of the fields

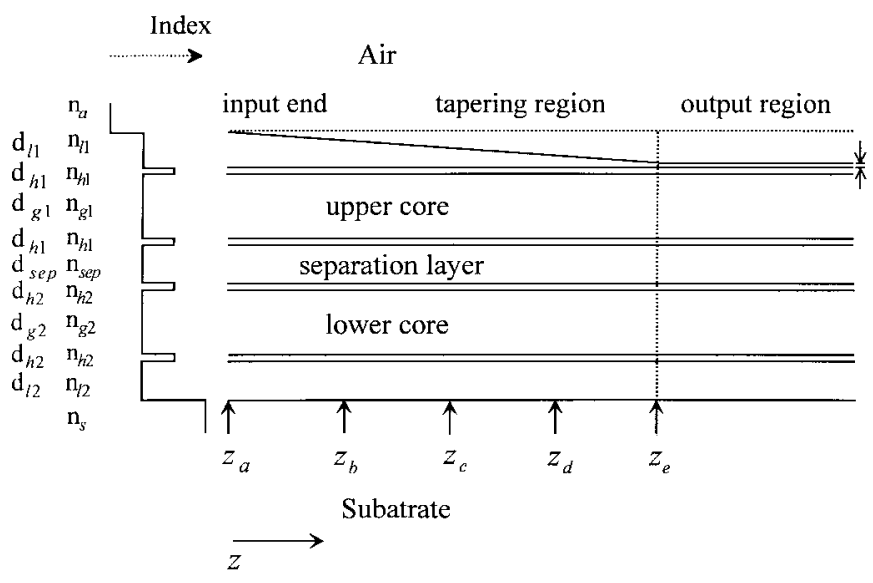

Fig. 1. The schematic view of an ARROW hybrid coupler.

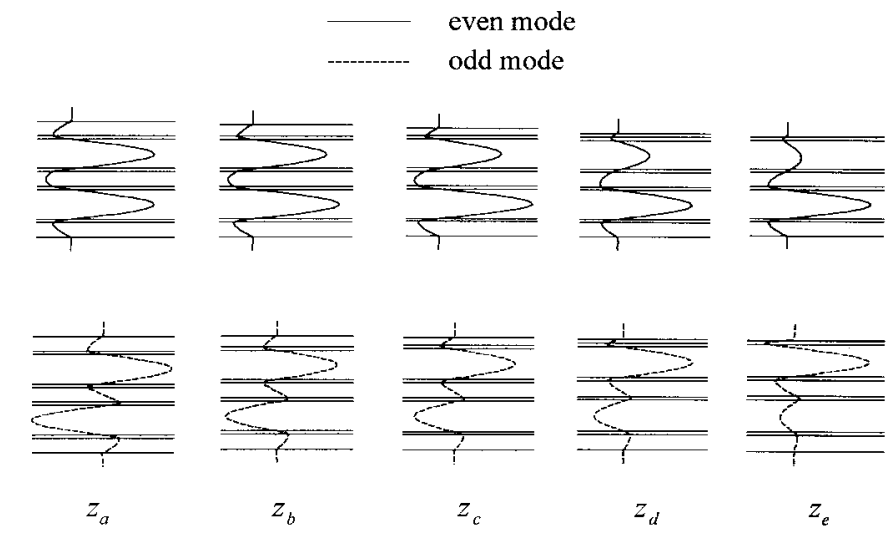

Fig. 2. The field distributions of two normal modes corresponding to the positions in Fig. 1.

for both modes in two individual waveguides are the same at the input end. The tapering angle is carefully designed to meet the adiabatic invariance condition such that the power resided in the initial modes at the beginning still remains in the same modes during traveling along the device [17]. The field of the even mode is mainly confined in the lower guide $g_{2}$ and the odd mode mainly in the upper guide $g_{1}$ at the output end, respectively. If two beams with the same amplitudes in phase are launched into two guides $g_{1}$ and $g_{2}$ at the input end, only the even mode can be excited and the power will be confined within guide $2\left(g_{2}\right)$ at the output end. On the other hand, when two input beams with the same amplitudes are out of phase, only the odd mode will be excited and the power will be confined within guide $1\left(g_{1}\right)$ at the output end as shown in Fig. 2. In such a way, the original power in two guide channels can be combined into one of these two guide channels depending on the phase difference between two input beams. In another case, if only single beam is launched into the input end of one of these two waveguides, even and odd modes are equally excited, and then adiabatically transferred into two guides ( $g_{1}$ and $g_{2}$ ), respectively, and the device functions as a power divider.

Based on the reciprocity theorem, a reversed hybrid coupler can convert the original power combiner to a power divider when only one beam is input to guide $1\left(g_{1}\right)$ or guide 


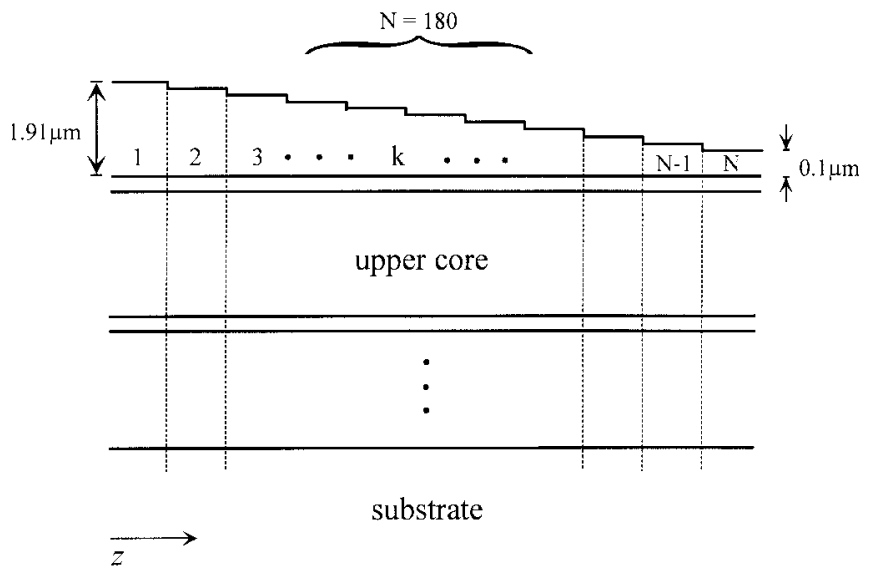

Fig. 3. The staircase model of an ARROW hybrid coupler.

$2\left(g_{2}\right)$. By combining these original and reversed hybrid couplers, an integrated-optical Michelson interferometer can be realized [2].

\section{StaIRCASE APPROXIMATION AND EIGENMODE EXPANSION}

In this section, staircase approximation and eigenmode expansion analysis are used to determine the adiabatic invariance condition [13]. In our analysis, the continuous tapered structure is divided into a series of staircase segments with small and abrupt discontinuities as shown in Fig. 3. The total number of segments is determined by satisfactory convergence when this number is enough increased. The field distributions and propagation losses of associated eigenmodes within each segment are obtained by applying the characteristic matrix method [9], [10]. The effective indexes of eigenmodes for our ARROW waveguides are solved in the complex plane due to their leaky characteristics. The imaginary parts of the effective indexes represent propagation losses of ARROW modes. The unphysical problem associated with the leaky mode representation can be relieved by the truncated method [18] with a suitable and systematic method to meet the orthogonality relation. Any field distribution $f(x, z)$ at any position within the $k$ th segment can be expressed by the summation of eigenmodes with their individual expansion coefficients as

$$
f^{(k)}(x, z)=\sum_{g=e, o} a_{g}^{(k)} u_{g}^{(k)}(x) \exp \left(-j \beta_{g}^{(k)} z\right)
$$

where $u_{g}^{(k)}(x), a_{g}^{(k)}$, and $\beta_{g}^{(k)}$ represent the normalized field distribution, propagation constant, and expansion coefficient of an eigenmode at the $k$ th segment, respectively, and subscript $g$ indicates a discrete guided mode which should be summed up. The normalized field distribution of the eigenmode $u_{g}^{(k)}(x)$ is multiplied by the phase term $\exp \left(-j \beta_{g}^{(k)} z\right)$ and its own expansion coefficient $a_{g}^{(k)}$ to express the wave propagation. In our case, only the first two modes, even and odd modes, are involved because only these modes can be efficiently excited by the fundamental mode from a single-mode fiber (or waveguide) input-coupling. The mode-conversion between these two modes can be simulated to determine the adiabatic invariance condition.
The boundary conditions at the step discontinuity give the relation as

$$
\begin{gathered}
\sum_{g=e, o} a_{g}^{(k)} u_{g}^{(k)}(x) \exp \left(-j \beta_{g}^{(k)} L^{(k)}\right) \\
=\sum_{g=e, o} a_{g}^{(k+1)} u_{g}^{(k+1)}(x)
\end{gathered}
$$

where $L^{(k)}$ is the length of the $k$ th segment. Here, it is assumed that the reflections of the next segments is negligible. This assumption is appropriate for our case since the fields of even and odd modes are mainly confined within the cores and only the thickness of the outermost cladding layer is changed. Combining the above equation with the orthogonal relations, the expansion coefficients of all modes at the $(k+1)$ th segment can be reconstructed as

$$
\begin{aligned}
a_{g}^{(k+1)}= & \sum_{h=e, o} a_{h}^{(k)} \exp \left(-j \beta_{g}^{(k)} L^{(k)}\right) \\
& \cdot \int u_{h}^{(k)}(x)\left[u_{g}^{(k+1)}(x)\right]^{*} d x \\
\equiv & \sum_{h=e, o} a_{h}^{(k)} \exp \left(-j \beta_{g}^{(k)} L^{(k)}\right)\left\langle u_{h}^{(k)}(x) \mid u_{g}^{(k+1)}(x)\right\rangle .
\end{aligned}
$$

Therefore, we obtain

$$
\boldsymbol{A}^{(k+1)}=\boldsymbol{A}^{(k)} \cdot \boldsymbol{P}^{(k)} \cdot \boldsymbol{C}^{(k, k+1)}
$$

where $\boldsymbol{A}^{(k)}$ is the input expansion matrix, which is composed of expansion coefficients of even and odd modes at the beginning of the $k$ th segment, i.e., $A^{(k)}=\left[a_{e} a_{o}\right]^{(k)} ; P^{(k)}$ describes the propagation phenomena of all modes in the $k$ th segment given as

$$
\boldsymbol{P}_{i j}^{(k)}=\left[\exp \left(-j \beta_{i}^{(k)} L^{(k)}\right)\right] \cdot \delta_{i j}
$$

where $\delta_{i j}$ is the Kronecker delta function and $C^{(k, k+1)}$ is the coupling matrix describing the coupling phenomena between modes in two adjacent segments at the corresponding step discontinuity, defined as

$$
C_{i j}^{(k, k+1)}=\left\langle u_{i}^{(k)}(x) \mid u_{j}^{(k+1)}(x)\right\rangle .
$$

Whenever the expansion matrix at the input end is determined, the output expansion matrix is obtained segment by segment from (6) as

$$
\begin{aligned}
\boldsymbol{A}^{(N)}=A^{(1)} \cdot[ & \boldsymbol{P}^{(1)} \cdot \boldsymbol{C}^{(1,2)} \cdot \boldsymbol{P}^{(2)} \cdot \boldsymbol{C}^{(2,3)} \cdots \\
& \left.\cdot \boldsymbol{C}^{(N-1, N)} \cdot \boldsymbol{P}^{(N)}\right] \equiv \boldsymbol{A}^{(1)} \cdot \boldsymbol{T}
\end{aligned}
$$

where $N$ is the total number of divided segments and $\boldsymbol{T}$ is the system transfer matrix. Once the tapered structure is modeled by the staircase approximation, the length of a segment $L^{(k)}$ can be changed when the tapering angle is varied, such that it is not necessary to recalculate the coupling matrices.

The mode-conversion effect can be observed by the phase behavior of even and odd modes. We can now extract the phase term in (9) to describe such effects. First, we observe the mode-conversion phenomena of an even mode. In this case, 
the input field is assumed to be the normalized even mode, i.e., $\boldsymbol{A}^{(1)}=\left[\begin{array}{ll}1 & 0\end{array}\right]$. If the adiabatic invariance condition holds, i.e., no cumulative mode conversion occurs, the mode coefficient $a_{o}$ at the output end will be very low. According to (5) and (6), the mode coefficient $a_{O}$ of the $(k+1)$ th segment can be represented as

$$
\begin{aligned}
a_{o}^{(k+1)}= & a_{e}^{(k)} \exp \left(-j \beta_{e}^{(k)} L^{(k)}\right) \cdot C_{e o}^{(k, k+1)} \\
& +a_{o}^{(k)} \exp \left(-j \beta_{o}^{(k)} L^{(k)}\right) \cdot C_{o o}^{(k, k+1)}
\end{aligned}
$$

Each variable of the above equation is separated into the amplitude term multiplied by the phase term as

$$
\begin{aligned}
\left|a_{o}^{(k+1)}\right| & \exp \left(j \phi_{o}^{(k+1)}\right) \\
= & {\left[\left|a_{e}^{(k)}\right| \exp \left(j \phi_{e}^{(k)}\right)\right] \exp \left(-j \beta_{e}^{(k)} L^{(k)}\right) } \\
& \cdot\left[\left|C_{e o}^{(k, k+1)}\right| \exp \left(j \phi_{C_{e o}}\right)\right] \\
+ & {\left[\left|a_{o}^{(k)}\right| \exp \left(j \phi_{o}^{(k)}\right)\right] \exp \left(-j \beta_{o}^{(k)} L^{(k)}\right) } \\
& \cdot\left[\left|C_{o o}^{(k, k+1)}\right| \exp \left(j \phi_{C_{o o}}\right)\right]
\end{aligned}
$$

where $\phi_{i}$ represents the corresponding phase term. Rearranging the above equation, we obtain

$$
\begin{aligned}
\left|a_{o}^{(k+1)}\right|= & \left|a_{e}^{(k)}\right| \cdot\left|C_{e o}^{(k, k+1)}\right| \cdot \exp \left(j \phi_{e o}\right) \\
& +\left|a_{o}^{(k)}\right| \cdot\left|C_{o o}^{(k, k+1)}\right| \cdot \exp \left(j \phi_{o o}\right)
\end{aligned}
$$

where $\phi_{e o}$ and $\phi_{o o}$ represent $\left(\phi_{e}^{(k)}-\beta_{e}^{(k)} L^{(k)}+\phi_{C_{e o}}-\phi_{o}^{(k+1)}\right)$ and $\left(\phi_{o}^{(k)}-\beta_{o}^{(k)} L^{(k)}+\phi_{C_{o o}}-\phi_{o}^{(k+1)}\right)$, respectively. The real part of (12) is expressed as

$$
\begin{aligned}
\left|a_{o}^{(k+1)}\right|= & \left|a_{e}^{(k)}\right| \cdot\left|C_{e o}^{(k, k+1)}\right| \cdot \cos \phi_{e o} \\
& +\left|a_{o}^{(k)}\right| \cdot\left|C_{o o}^{(k, k+1)}\right| \cdot \cos \phi_{o o} .
\end{aligned}
$$

Thus, the degree of mode conversion can be observed by the phase behavior as described in (13) [13]. If the phase term $\cos \phi_{e o}$ is locked near 1 or -1 especially in the region where the coupling coefficient $\left|C_{e o}^{(k, k+1)}\right|$ is relatively large along the propagation direction, substantial mode conversion from the even mode to the odd mode will occur. On the contrary, if the phase term oscillates rapidly across zero, $\left|a_{o}^{(k)}\right|$ will not cumulatively increase and mode conversion does not occur. The above analysis is also valid when the input field is an odd mode.

\section{Design Example and Simulation Results}

In this section, our design example is based on a $\mathrm{Si}$ substrate $\left(n_{s}=3.5\right)$ and the operating wavelength is $\lambda=$ $0.6328 \mu \mathrm{m} . \mathrm{SiO}_{2}$ with a refractive index of 1.46 is used for guiding layers, the separation layer and low-index cladding layers, i.e., $n_{g_{1}}=n_{g_{2}}=n_{\text {sep }}=n_{l_{1}}=n_{l_{2}}=1.46$, and the glass mixture of $\mathrm{BaO}$ and $\mathrm{SiO}_{2}$ with a refractive index of 1.50 is used as high-index cladding layers, i.e., $n_{h_{1}}=n_{h_{2}}=1.5$.

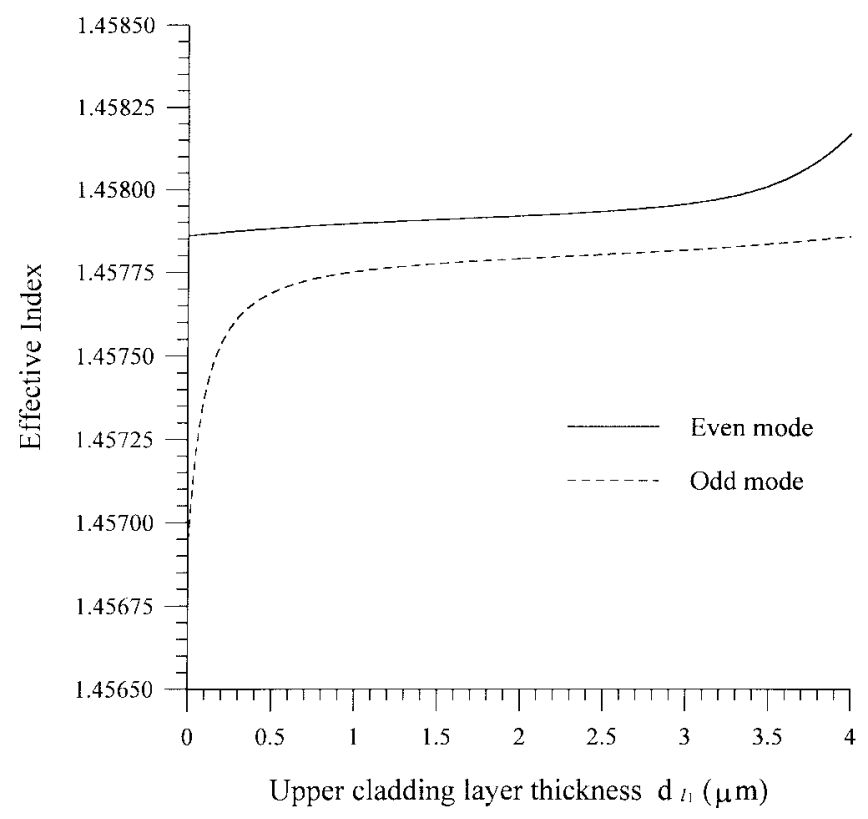

Fig. 4. The dispersion relation versus the thickness of the upper cladding layer $d_{l_{1}}$. The parameters are $n_{g_{1}}=n_{g_{2}}=n_{\text {sep }}=n_{l_{1}}=n_{l_{2}}=1.46$, $n_{h_{1}}=n_{h_{2}}=1.5, d_{g_{1}}=d_{g_{2}}=4 \mu \mathrm{m}, d_{\text {sep }}=d_{l_{2}}=2 \mu \mathrm{m}$, $d_{h_{1}}=d_{h_{2}}=0.448 \mu \mathrm{m}$ and $\lambda=0.6328 \mu \mathrm{m}$.

The upper waveguide thickness $d_{g_{1}}$ is $4 \mu \mathrm{m}$ such that this large core is compatible with single-mode optical fibers [9], [10]. Based on (1) and (2) we obtain $d_{g_{2}}=4 \mu \mathrm{m}, d_{l_{2}}=2 \mu \mathrm{m}$, and $d_{h_{1}}=d_{h_{2}}=0.448 \mu \mathrm{m}$ for $\lambda=0.6328 \mu \mathrm{m}$. Also based on (2) the thickness of the separation layer can be adjusted as $d_{\text {sep }}=2 \mu \mathrm{m} \times\left(2 P_{j}+1\right)$ for easy alignment to optical fibers. In our analysis the minimum value of $d_{\text {sep }}=2 \mu \mathrm{m}$ is chosen for numerical convenience. The effective indexes of even and odd modes as functions of the upper cladding thickness are illustrated in Fig. 4. We then determine the thickness of the upper cladding layer, which will influence the symmetric degree of the dual ARROW waveguides. From the dispersion results shown in Fig. 4, it can be seen that, in the symmetric region, i.e., $d_{l_{1}}=d_{l_{2}}$, both even and odd modes are described by nondispersive curves which imply symmetric field distributions for two modes, and optical power can efficiently exchange between two ARROW waveguides. In the asymmetric region, i.e., $d_{l_{1}}$ approach zero, the dispersive curves imply asymmetric field distributions for two modes. The maximum coupling efficiency as a function of the upper cladding thickness is shown in Fig. 5 [10]. It is observed that efficient coupling occurs in a symmetric structure and weak coupling in an asymmetric structure. Since our ARROW hybrid coupler is tapered from an exactly symmetric structure to an extremely asymmetric structure, we choose the initial value of $d_{l_{1}}$ to be $1.91 \mu \mathrm{m}$ such that the maximum coupling efficiency is high and both the even mode and odd modes are equally distributed into two cores, and the final value of $d_{l_{1}}$ to be $0.1 \mu \mathrm{m}$ such that the maximum coupling efficiency is sufficiently low and both modes reside mainly in two individual cores. The mode conversion along the whole device is then analyzed by eigenmode propagation as described in the previous section to find the optimized tapering angle. In our 


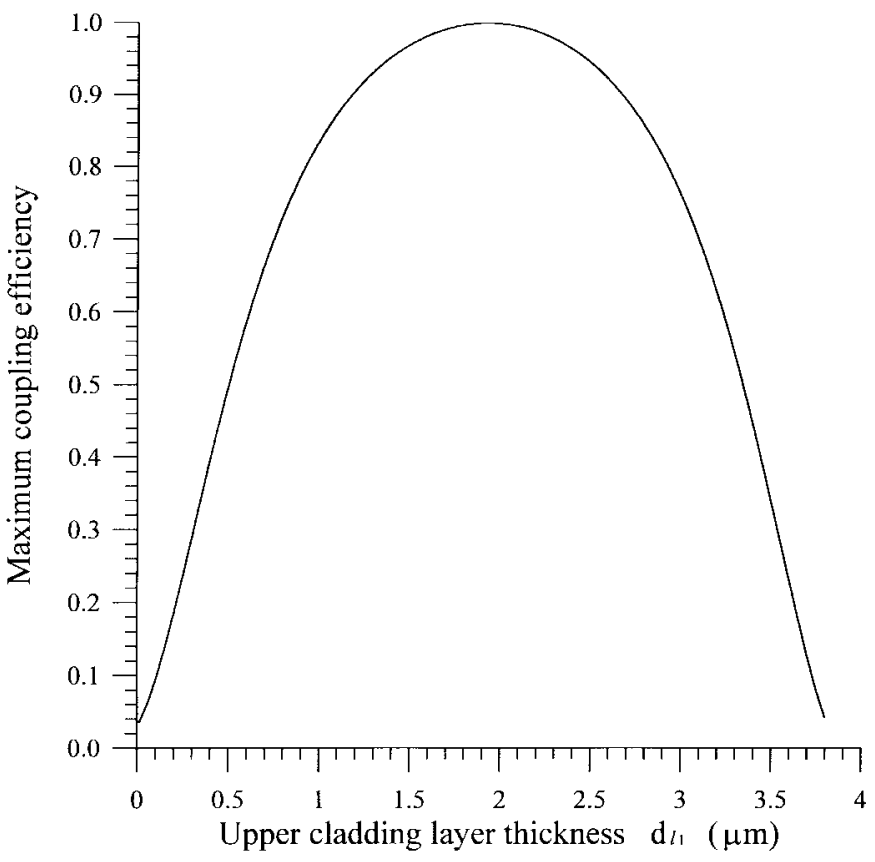

Fig. 5. Maximum coupling efficiency as a function of the upper cladding layer thickness $d_{l_{1}}$. The parameters are $n_{g_{1}}=n_{g_{2}}=$ $n_{\mathrm{sep}}=n_{l_{1}}=n_{l_{2}}=1.46, n_{h_{1}}=n_{h_{2}}=1.5, d_{g_{1}}=d_{g_{2}}=4 \mu \mathrm{m}$, $d_{\text {sep }}=d_{l_{2}}=2 \mu \mathrm{m}, d_{h_{1}}=d_{h_{2}}=0.448 \mu \mathrm{m}$, and $\lambda=0.6328 \mu \mathrm{m}$.

analysis, the continuous structure is divided into $N$ sections with small abrupt steps as shown in Fig. 3. The length of each section $L^{(k)}$ as a function of the tapering angle is

$$
L^{(k)}=\frac{\Delta d_{l_{1}}}{N \cdot \tan \alpha}
$$

where $\Delta d_{l_{1}}$ is the total tapered thickness of the upper cladding layer and $\alpha$ is the tapering angle. In this case, it is found that $N=180$ is sufficiently large to describe the mode conversion effect of the original continuous structure. As described in the previous section, the input field is assumed to be the normalized even mode, i.e., $\boldsymbol{A}^{(1)}=\left[\begin{array}{ll}1 & 0\end{array}\right]$. Fig. 6 shows the mode coefficients $a_{e}$ and $a_{o}$ as functions of $d_{l_{1}}$ when the input beam is propagating along the whole device for various tapering angles. The proper tapering angle is chosen to avoid mode conversion between two normal modes and prevent two normal modes from conversion into radiation modes. In Fig. 6, it is observed that a substantial mode conversion from the even mode to the odd mode occurs when $\alpha=0.05^{\circ}$, whereas nearly adiabatic invariance is achieved when $\alpha=0.03^{\circ}$. Fig. 7 shows the phase term $\cos \phi_{e o}$ in (13) versus $d_{l_{1}}$ for various tapering angles. The coupling coefficients $C_{e o}$ are simultaneously shown in this figure. It is found that the phase term oscillates rapidly in the case of $\alpha=0.01^{\circ}$, whereas it is locked near -1 in the region of a large $C_{e o}$ when $\alpha=0.05^{\circ}$. In comparison with Figs. 6 and 7, $a_{o}$ oscillates as $\cos \phi_{e o}$ does when $\alpha=0.01^{\circ}$, and thus no cumulative mode conversion occurs. On the other hand, the cumulative mode conversion is serious when $\alpha=0.05^{\circ}$. In Fig. 8, the mode expansion coefficients at the output end for $0.001^{\circ}<\alpha<0.1^{\circ}$ are given. It can be seen that $a_{e}$ decreases dramatically as $\alpha$ becomes very small because the radiation loss of the ARROW modes into substrate increases as the device length is very long.

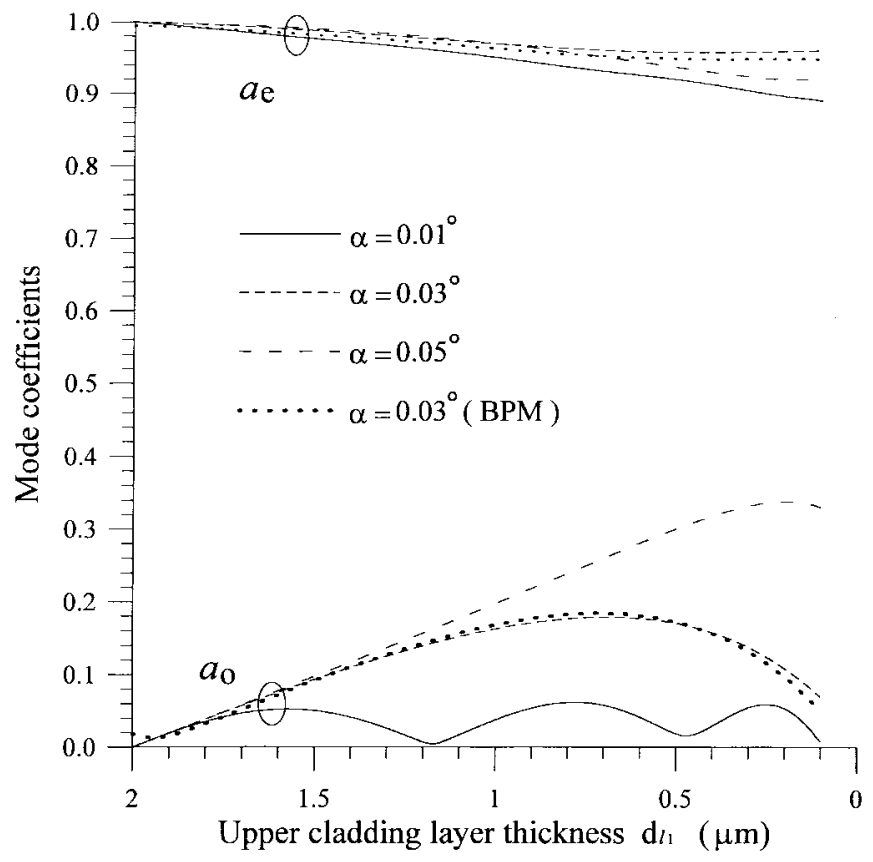

Fig. 6. The mode coefficients $a_{e}$ and $a_{o}$ as functions of the thickness of the upper cladding layer for the tapering angles $\alpha=0.01,0.03$, and $0.05^{\circ}$. The input field is an even mode. BPM simulation results for $\alpha=0.03^{\circ}$ are also given for comparison. The parameters are $n_{g_{1}}=n_{g_{2}}=n_{\mathrm{sep}}=n_{l_{1}}=$ $n_{l_{2}}=1.46, n_{h_{1}}=n_{h_{2}}=1.5, d_{g_{1}}=d_{g_{2}}=4 \mu \mathrm{m}, d_{\mathrm{sep}}=d_{l_{2}}=2 \mu \mathrm{m}$, $d_{h_{1}}=d_{h_{2}}=0.448 \mu \mathrm{m}$, and $\lambda=0.6328 \mu \mathrm{m}$.

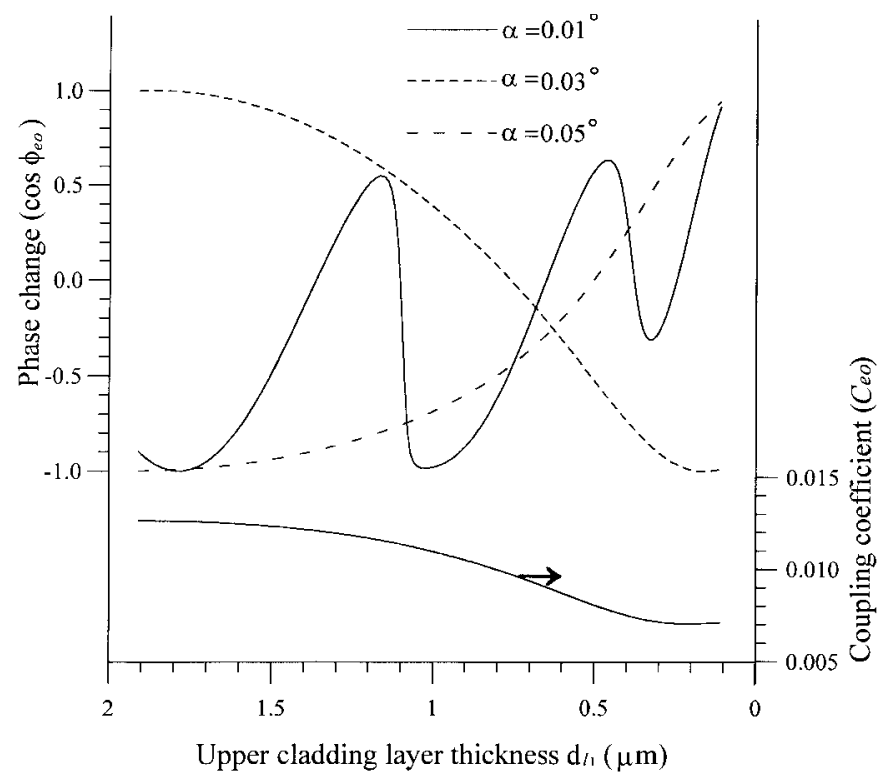

Fig. 7. The phase behavior associated with Fig. 6.

However, the mode conversion occurs when $\alpha$ becomes large as mentioned. The optimum tapering angle is chosen as $0.03^{\circ}$ for this device.

The beam propagation method (BPM) with a transparent boundary condition (TBC) and an efficient interface condition is also used to verify our design and analysis [14]-[16]. An even mode with a unit power is launched into our ARROW hybrid coupler with $\alpha=0.03^{\circ}$, and the overlap integral between the calculated field and the eigenmode fields (one 


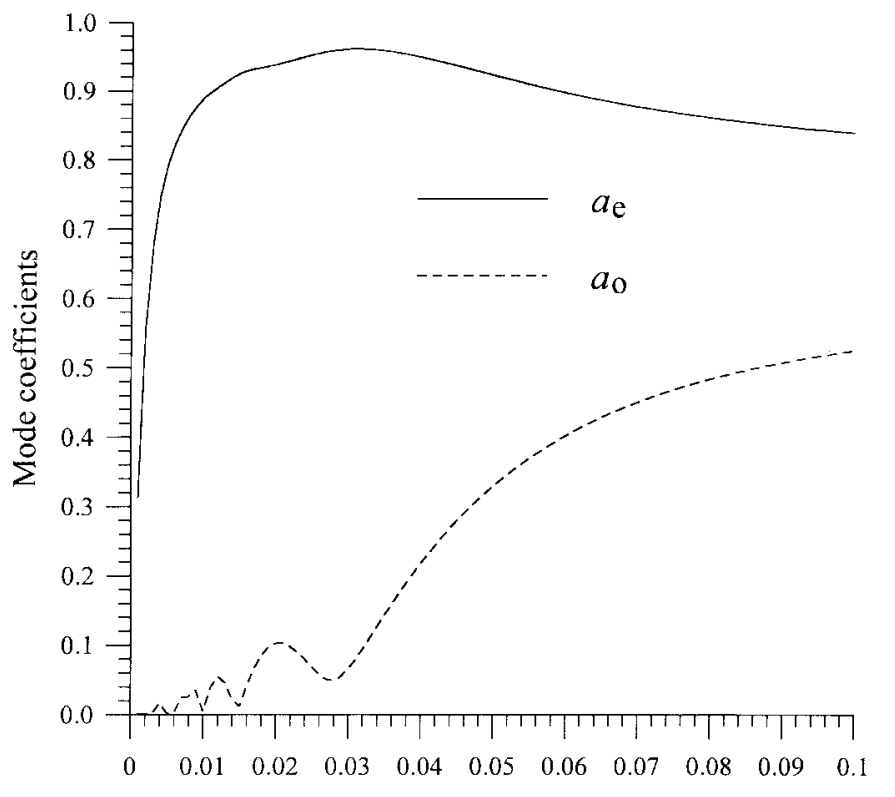

Tapering angle $\alpha$ (deg)

Fig. 8. The cumulative mode coefficients at the end of this device as functions of the tapering angle. The parameters are $n_{g_{1}}=n_{g_{2}}=$ $n_{\mathrm{sep}}=n_{l_{1}}=n_{l_{2}}=1.46, n_{h_{1}}=n_{h_{2}}=1.5, d_{g_{1}}=d_{g_{2}}=4 \mu \mathrm{m}$, $d_{\mathrm{sep}}=d_{l_{2}}=2 \mu \mathrm{m}, d_{h_{1}}=d_{h_{2}}=0.448 \mu \mathrm{m}, d_{l_{1}}$ is tapered from $1.91 \mu \mathrm{m}$ to $0.01 \mu \mathrm{m}, \alpha=0.03^{\circ}$, and $\lambda=0.6328 \mu \mathrm{m}$.

even and one odd modes) along the propagation direction is plotted in Fig. 6 as well. It is shown that BPM simulation results agree with the results of our previous analysis. The field evolution profiles with the input field of an even mode and an odd mode are shown in Fig. 9(a) and (b), respectively. To evaluate the performance of our hybrid coupler, the extinction ratios for two modes are defined as

$$
\begin{aligned}
\xi_{o, e}= & 10 \log _{10} \frac{P_{o} \text { at the output end }}{P_{e} \text { at the input end }}, \\
& \text { with the even mode as the input field } \\
\xi_{e, o}= & 10 \log _{10} \frac{P_{e} \text { at the output end }}{P_{o} \text { at the input end }} \\
& \text { with the odd mode as the input field }
\end{aligned}
$$

where $P_{e}$ and $P_{o}$ are optical power of the even mode and the odd mode, respectively. The extinction ratios $\xi_{e, o}$ and $\xi_{o, e}$ should be very small when the device is nearly adiabatic and radiation losses are small. In our case, $\xi_{o, e}=-23.10 \mathrm{~dB}$ and $\xi_{e, o}=-22.87 \mathrm{~dB}$. The radiation losses are $0.38 \mathrm{~dB}$ and $0.31 \mathrm{~dB}$, respectively, as desired. Finally, further simulations with a unit-power even mode and odd mode as the input field from the original output end are performed to verify the reciprocity law as shown in Fig. 10(a) and (b), respectively. The extinction ratios of $\xi_{o, e}=-23.05 \mathrm{~dB}$ and $\xi_{e, o}=-24.30$ $\mathrm{dB}$ are obtained, and the radiation losses are 0.34 and 0.25 $\mathrm{dB}$, respectively. All these results verify the function of our devices.

\section{CONCLUSION}

A novel hybrid coupler based on antiresonant reflecting optical waveguides (ARROW's) is proposed. This ARROW

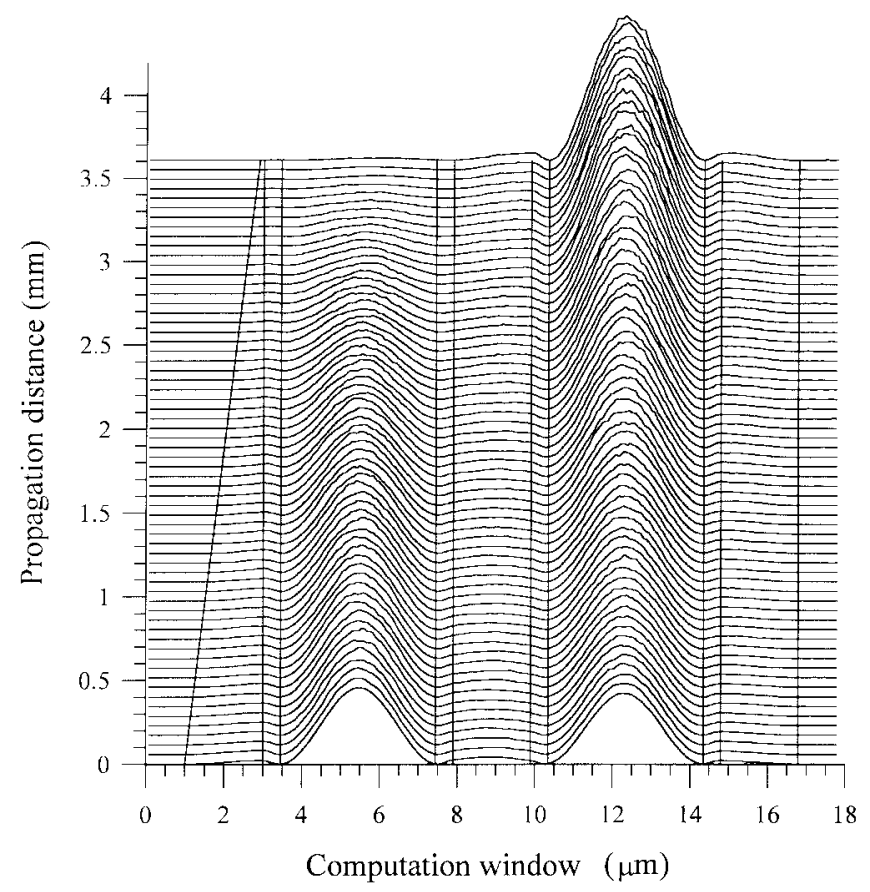

(a)

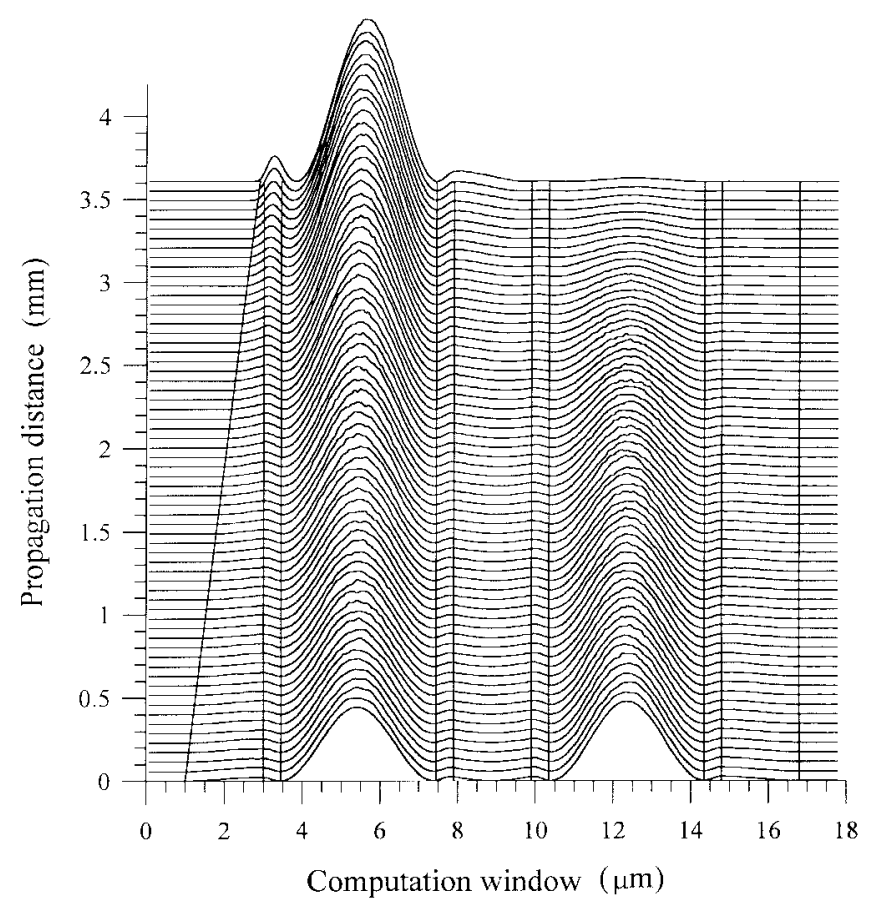

(b)

Fig. 9. The power evolution profile with an input field of a normal mode along the propagation distance for an ARROW hybrid coupler. The parameters are $n_{g_{1}}=n_{g_{2}}=n_{\text {sep }}=n_{l_{1}}=n_{l_{2}}=1.46, n_{h_{1}}=n_{h_{2}}=1.5$, $d_{g_{1}}=d_{g_{2}}=4 \mu \mathrm{m}, d_{\mathrm{sep}}=d_{l_{2}}=2 \mu \mathrm{m}, d_{h_{1}}=d_{h_{2}}=0.448 \mu \mathrm{m}, d_{l_{1}}$ is tapered from $1.91 \mu \mathrm{m}$ to $0.01 \mu \mathrm{m}, \alpha=0.03^{\circ}$, and $\lambda=0.6328 \mu \mathrm{m}$. (a) An even mode input and (b) an odd mode input.

hybrid coupler consists of two parallel antiresonant reflecting optical waveguides with a tapered outermost cladding layer. Compatible core dimensions and indexes with singlemode fibers and a large separation thickness between two waveguide channels of an ARROW hybrid coupler can be 


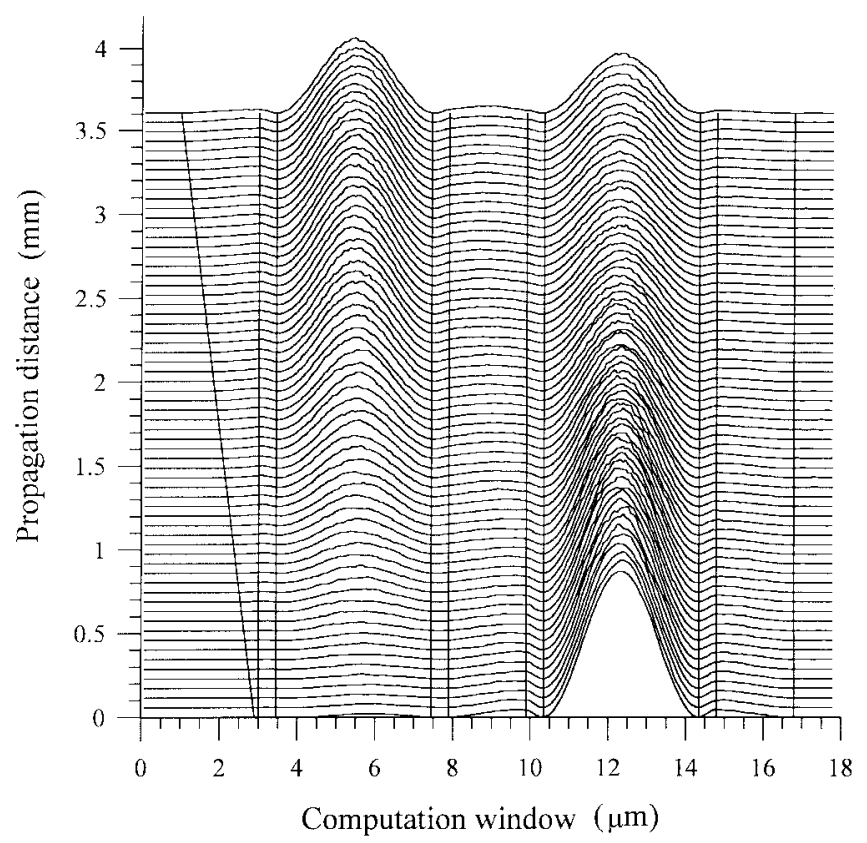

(a)

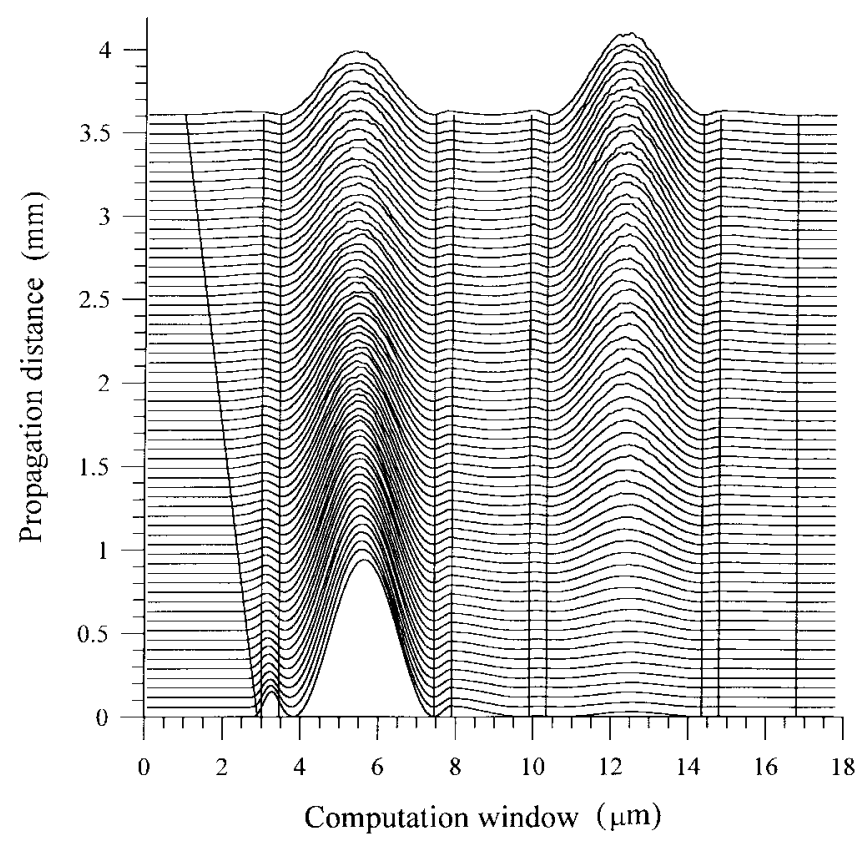

(b)

Fig. 10. The power evolution profile with an input field of a normal mode along the propagation distance for a reversed ARROW hybrid coupler. The parameters are $n_{g_{1}}=n_{g_{2}}=n_{\text {sep }}=n_{l_{1}}=n_{l_{2}}=1.46, n_{h_{1}}=n_{h_{2}}=1.5$, $d_{g_{1}}=d_{g_{2}}=4 \mu \mathrm{m}, d_{\text {sep }}=d_{l_{2}}=2 \mu \mathrm{m}, d_{h_{1}}=d_{h_{2}}=0.448 \mu \mathrm{m}, d_{l_{1}}$ is increased from $0.01 \mu \mathrm{m}$ to $1.91 \mu \mathrm{m}, \alpha=0.03^{\circ}$, and $\lambda=0.6328 \mu \mathrm{m}$. (a) An even mode input and (b) an odd mode input.

achieved by utilizing the periodic property of the antiresonant conditions. The input and output coupling efficiency are thus significantly improved compared to a conventional waveguide hybrid coupler. With an optimum design of the outermost cladding layer thickness, equal excitations for two normal modes (one even and one odd modes) at input end can be realized and the crosstalk at the output end is low. In addition, the surface scattering is relieved because only the thickness of outermost cladding layer is gradually changed. The tapering angle is designed to ensure the adiabatic invariance condition. Eigenmode expansion analysis is used to design this device and the beam propagation method is used to verify the device characteristics. A design example based on a $\mathrm{Si}$-substrate is given. The extinction ratios of -23.10 and $-22.87 \mathrm{~dB}$ with an input for an even mode and an odd mode are obtained, respectively, and the corresponding radiation losses of 0.38 and $0.31 \mathrm{~dB}$ are also obtained. This versatile optical device can be efficiently applied to optical communications, optical signal processing, and optical microsensing systems.

\section{ACKNOWLEDGMENT}

The authors would like to thank the reviewers for their helpful suggestions.

\section{REFERENCES}

[1] M. Izutsu, A. Enokihara, and T. Sueta, "Optical hybrid coupler," Opt. Lett., vol. 7, no. 11, pp. 549-551, 1982.

[2] _ , "Optical-waveguide micro-displacement sensor," Electron. Lett., vol. 18 , no. 20 , pp. 867-868, 1982.

[3] C. P. Hussel, R. V. Ramaswamy, R. Srivastava, and J. L. Jackel, "Wavelength and polarization insensitive $3 \mathrm{~dB}$ cross-coupler power divider by ion exchange in glass," Appl. Phys. Lett., vol. 56, no. 24 pp. 2381-2383, 1990.

[4] A. Tervonen, P. Pöyhönen, S. Honkanen, and M. Tahkokorpi, "A guided-wave Mach-Zehnder interferometer structure for wavelength multiplexing," IEEE Photon. Technol. Lett., vol. 3, pp. 516-518, June 1991.

[5] W. J. Wang, S. Honkanen, S. I. Najafi, and A. Tervonen, "Four port guided-wave nonsymmetric Mach-Zehnder interferometer," Appl. Phys. Lett., vol. 61, no. 2, pp. 150-152, 1992.

[6] H. Feng, X. Li, Z. Yang, and M. Wang, " $2 \times 2$ GaAs asymmetric Mach-Zehnder interferometer switch," Appl. Phys. Lett., vol. 60, no. 23, pp. 2843-2845, 1992.

[7] M. A. Duguay, Y. Kokubun, and T. L. Koch, "Antiresonant reflecting optical waveguides in $\mathrm{SiO}_{2}$-Si multilayer structures," Appl. Phys. Lett., vol. 49, no. 1, pp. 13-15, 1986.

[8] T. Baba, Y. Kokubun, T. Sakaki, and K. Iga, "Loss reduction of an ARROW waveguide in shorter wavelength and its stack configuration," J. Lightwave Technol., vol. 6, pp. 1440-1444, Sept. 1988

[9] M. Mann, U. Trutschel, C. Wachter, L. Leine, and F. Lederer, "Directional coupler based on an antiresonant reflecting optical waveguide," Opt. Lett., vol. 16, no. 11, pp. 805-807, 1991.

[10] Y.-H. Chen and Y.-T. Huang, "Coupling efficiency analysis and control of dual antiresonant reflecting optical waveguides," J. Lightwave Technol., vol. 14, pp. 1507-1513, June 1996.

[11] J.-J. Deng and Y.-T. Huang, "A novel hybrid coupler based on antiresonant reflecting optical waveguides," in Proc. IEEE CLEO/Europe-EQEC '96, Hamburg, Germany, Sept. 1996.

[12] D. Marcuse, "Radiation losses of tapered dielectric slab waveguides," Bell Syst. Tech. J., vol. 49, no. 2, pp. 273-290, 1970.

[13] W. K. Burns and A. F. Milton, "Mode conversion in planar-dielectric separating waveguides," IEEE J. Quantum Electron., vol. QE-11, pp. 32-39, Jan. 1975.

[14] Y. Chung and N. Dagli, "An assessment of finite different beam propagation method," IEEE J. Quantum Electron., vol. 26, pp. 1335-1339, Aug. 1990.

[15] G. R. Hadley, "Transparent boundary condition for beam propagation," Opt. Lett., vol. 16, no. 9, pp. 624-626, 1991.

[16] H. J. W. H. Hoekstra, G. J. M. Krijnen, and P. V. Lambeck, "Efficient interface conditions for the finite difference beam propagation method," J. Lightwave Technol., vol. 10, pp. 1352-1355, Oct. 1992.

[17] Y. Shani, C. H. Henry, R. C. Kistler, R. F. Kazarinov, and K. J. Orlowsky, "Integrated optic adiabatic devices in silicon," IEEE $J$. Quantum Electron., vol. 27, pp. 556-566, Mar. 1991.

[18] R. Sammut and A. W. Snyder, "Leaky modes on a dielectric waveguide: Orthogonality and excitation," Appl. Opt., vol. 15, no. 14, pp. 1040-1044, 1976. 


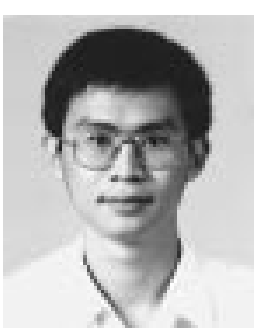

Jau-Jan Deng (S'93) received the B.S. degrees in electronics from the National Chiao-Tung University, Taiwan, in 1992 . He is currently pursuing the Ph.D. degree.

His current interests in research are integratedoptic devices for optical interconnects, optical communications, and optical sensors.

Mr. Deng is a member of the Optical Society of America (OSA).

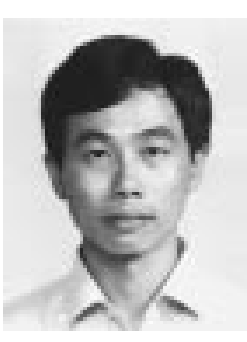

Yang-Tung Huang (M'82) received the B.S. degree in electrophysics and the M.S. degree in electronics from National Chiao-Tung University, Taiwan, in 1978 and 1982, respectively, and the Ph.D. degree in electrical and computer engineering from the University of Arizona, Tucson, in 1990.

In 1983, he joined the Faculty at National ChiaoTung University, where he is currently a Professor at the Department of Electronics Engineering and the Institute of Electronics and also Director of the Institute of Electronics. His current research interests include integrated optics, holography, and optical interconnects.

Dr. Huang is a member of the Optical Society of America (OSA). 